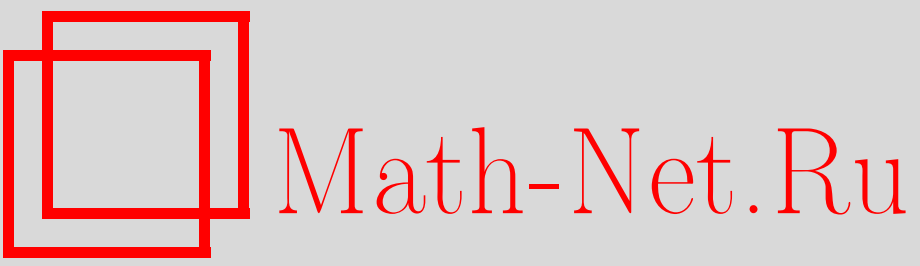

Г. С. Шаров, Классификация ротационных движений для струнной модели бариона "треугольник", ТМФ, 1998, том 114, номер 2, 277-295

DOI: https://doi.org/10.4213/tmf840

Использование Общероссийского математического портала Math-Net.Ru подразумевает, что вы прочитали и согласны с пользовательским соглашением

http://www.mathnet.ru/rus/agreement

Параметры загрузки:

IP: 35.173 .219 .149

26 апреля 2023 г., 14:57:30 
ТЕОРЕТИЧЕСКАЯ

И МАТЕМАТИЧЕСКАЯ

ФИЗИКА

Том 114, № 2

февраль, 1998

(C) $\mathbf{1 9 9 8} \mathrm{r}$.

Г. С. Шаров*

\section{КЛАССИФИКАЦИЯ РОТАЦИОННЫХ ДВИЖЕНИЙ ДЛЯ СТРУННОЙ МОДЕЛИ БАРИОНА “ТРЕУГОЛЬНИК”}

Рассматривается модель бариона, в которой три материальные точки (три кварка) попарно соединены релятивистскими струнами, образующими криволинейный треугольник. Для этой модели найдены и изучены классические аналитические решения, отвечающие плоскому равномерному вращению системы в случае произвольных масс кварков $m_{i}$. Сечения соответствующих мировых поверхностей плоскостью $t=\mathrm{const}$ являются криволинейными треугольниками, составленными из отрезков гипоциклоиды. Проведена полная классификация движений данного вида, различающихся топологически своей конфигурацией, а также наличием и числом внутренних безмассовых точек струны, движущихся со скоростью света. В основу классификации положено исследование предельных состояний системы при $m_{i} \rightarrow 0$. Рассчитанная зависимость углового момента исследуемых движений от энергии открывает возможность моделирования с их помощью барионных состояний на реджевских траекториях.

\section{ВВЕДЕНИЕ}

Рассматриваемая здесь струнная модель бариона "треугольник" находится в генетической связи с моделью релятивистской струны с массами на концах, которая описывает мезон - состояшую из кварка и антикварка систему, связанную сильным взаимодействием $[1,2]$. В этой модели мезона два точечных массивных кварка соединены одномерной протяженной релятивистской струной, закон движения которой определяется условием экстремальности плошади поверхности (мировой поверхности), заметаемой этой струной в пространстве Минковского. Струнная модель мезона обеспечивает согласующийся с квантовой хромодинамикой линейно растуший потеншиал межкваркового взаимодействия $[1,3]$ и дает возможность описания мезонных состояний на траекториях Редже [4].

Вслед за мезонными были предложены в нескольких вариантах струнные модели бариона - трехкваркового связанного состояния $[1,5,6]$. При построении этих моделей на классическом уровне необходимо выбрать геометрический характер соединения трех материальных точек релятивистскими струнами. Возможны варианты: а) модель, в которой данные точки соединены двумя отрезками струны, связываюшими соответствен-

\footnotetext{
${ }^{*}$ Тверской государственный университет, Тверь, Россия
} 
но первый кварк со вторым и второй с третьим; б) модель, в которой три идущих от кварков струны соединяются в четвертой (безмассовой) точке; в) модель “треугольник" .

K настояшему времени подробно разрабатывался лишь вариант “б”, в частности в работах [6]. Модель "а" описана лиш качественно в [5].

В [7] и в продолжающей ее настояшей работе рассматривается струнная модель бариона “треугольник", которая практически еще не исследована. В этой модели три материальные точки (три кварка) попарно соединены релятивистскими струнами, образуя в каждый момент времени криволинейный треугольник в пространстве. Если натяжения всех трех струн равны, то этот объект можно воспринимать как замкнутую струну, нагруженную тремя точечными массами. С точки зрения описания взаимодействия кварков в барионе данная модель выглядит наиболее естественно среди упомянутых выше.

При переходе от струнной модели мезона к модели бариона “треугольник” возникают дополнительные трудности, связанные, в частности, со следующими обстоятельствами. Мировая поверхность струны в этой модели имеет разрывы производных на траекториях кварков. Для произвольного движения невозможно задать параметризацию, в которой все эти траектории являются координатными линиями, а индуцированная метрика на мировой поверхности - конформно-плоской. Пространственноподобные координатные линии, вообще говоря, не замкнуты.

В настоящей работе эти трудности преодолены для некоторого класса движений системы. В разделе 1 приведены уравнения движения и краевые условия (условия на траекториях кварков) для данной модели. В разделах 2 и 3 найдены и исследованы решения этих уравнений, отвечаюшие равномерному врашению системы относительно центра масс в некоторой плоскости. В разделе 4 проведена классификация таких решений. В разделе 5 с помощью энергии и момента, вычисленных для найденных движений, исследуются возможности описания барионных состояний на траекториях Редже.

\section{1. УРАВНЕНИЯ ДВИЖЕНИЯ И УСЛОВИЯ НА ТРАЕКТОРИЯХ КВАРКОВ}

Мы будем рассматривать модель бариона “треугольник" как замкнутую релятивистскую струну с натяжением $\gamma$, нагруженную тремя точечными массами $m_{1}, m_{2}, m_{3}$. Действие для этой системы имеет вид [7]

$$
S=-\int_{\tau_{1}}^{\tau_{2}} d \tau\left\{\gamma \int_{\sigma_{0}(\tau)}^{\sigma_{3}(\tau)}\left[\left(\dot{X} X^{\prime}\right)^{2}-\dot{X}^{2} X^{\prime 2}\right]^{\frac{1}{2}} d \sigma+\sum_{i=1}^{3} m_{i} \sqrt{\left[\frac{d}{d \tau} X^{\mu}\left(\tau, \sigma_{i}(\tau)\right)\right]^{2}}\right\}
$$

Здесь $X^{\mu}(\tau, \sigma)$ - координаты точки струны в $N$-мерном пространстве Минковского, скорость света $c=1, \quad(a b)=a^{\mu} b_{\mu}-\left(\right.$ псевдо)скалярное произведение, $\dot{X}^{\mu}=\partial_{\tau} X^{\mu}$, $X^{\prime \mu}=\partial_{\sigma} X^{\mu}, \sigma_{i}(\tau), i=0,1,2,3,-$ внутренние координаты мировых линий кварков. Уравнения $\sigma=\sigma_{0}(\tau)$ и $\sigma=\sigma_{3}(\tau)$ определяют траекторию одного и того же (третьего по счету) кварка, что отражает факт замкнутости струны и в общем виде может быть записано следующим образом:

$$
X^{\mu}\left(\tau, \sigma_{0}(\tau)\right)=X^{\mu}\left(\tau^{*}, \sigma_{3}\left(\tau^{*}\right)\right) .
$$


Подчеркнем, что параметры $\tau$ и $\tau^{*}$, фигурируюшие в этих двух параметризациях одной и той же линии, вообще говоря, не совпадают. Это означает, что координатные линии $\tau=$ const на мировой поверхности не замкнуты, они начинаются (при $\left.\sigma=\sigma_{0}\right)$ и заканчиваются (при $\sigma=\sigma_{3}$ ) в различных точках поверхности и пространства Минковского.

Равенство $\tau=\tau^{*}$ имеет место лишь при специальном выборе параметров $\tau$ и $\sigma$, в частности при $\tau=t \equiv X^{0}$.

Функция $X^{\mu}(\tau, \sigma)$ на линиях $\sigma=\sigma_{i}(\tau)$ может иметь разрывы производных (за исключением тангенциальных к данным линиям).

Уравнения движения и краевые условия для данной модели выводятся в работе [7] путем варьирования и минимизации действия (1.1). С помощью невырожденной замены координат $\tau=\tau(\tilde{\tau}, \tilde{\sigma}), \sigma=\sigma(\tilde{\tau}, \tilde{\sigma})$, оставляющей инвариантным действие $(1.1)$, индуцированную метрику на всей мировой поверхности всегда можно сделать непрерывной и привести к конформно-плоскому виду [7], другими словами, ввести координаты, в которых выполнены условия ортонормальности

$$
\dot{X}^{2}+X^{\prime 2}=0, \quad\left(\dot{X} X^{\prime}\right)=0
$$

В этих координатах принимают простейший вид уравнения движения

$$
\ddot{X}^{\mu}-X^{\prime \prime \mu}=0
$$

и краевые условия

$$
\begin{gathered}
\left.m_{i} \frac{d}{d \tau} \frac{\dot{X}^{\mu}+\sigma_{i}^{\prime}(\tau) X^{\prime \mu}}{\sqrt{\dot{X}^{2} \cdot\left(1-\sigma_{i}^{\prime 2}\right)}}\right|_{\sigma=\sigma_{i}(\tau)}-\left.\gamma\left(X^{\prime \mu}+\sigma_{i}^{\prime}(\tau) \dot{X}^{\mu}\right)\right|_{\sigma=\sigma_{i}+0}+ \\
+\left.\gamma\left(X^{\prime \mu}+\sigma_{i}^{\prime}(\tau) \dot{X}^{\mu}\right)\right|_{\sigma=\sigma_{i}-0}=0, \quad i=1,2,3 .
\end{gathered}
$$

Для третьего кварка $(i=3)$ в первых двух слагаемых условия (1.5) следует положить $\sigma=\sigma_{0}(\tau)$, а в последнем $-\sigma=\sigma_{3}\left(\tau^{*}\right)$ и заменить $\tau$ на $\tau^{*}$, если условие замыкания имеет вид (1.2).

По своему физическому смыслу краевые условия (1.5) являются уравнениями движения (вторым законом Ньютона) для каждой из трех материальных точек, причем действуюшие на них силы натяжения со стороны двух отрезков струны по модулю равны $\gamma$.

С помошью невырожденной замены координат $\tau \pm \sigma=f_{ \pm}(\tilde{\tau} \pm \tilde{\sigma})$ [1], оставляюшей инвариантными условия (1.3) и уравнения (1.4), (1.5), можно зафиксировать две из четырех функций $\sigma_{i}(\tau)$, положив, например,

$$
\sigma_{1}(\tau)=0, \quad \sigma_{2}(\tau)=\pi
$$

Выполнения первого из условий (1.6) можно добиться выбором функции $f_{+}(\eta)$, оставляя тождественной $f_{-}(\eta)=\eta$, выполнения второго - еше одной заменой с $f_{+}=f_{-}$. 
После этого в нашем распоряжении останутся лишь сохраняюшие (1.6) репараметризации с $f_{+}(\eta)=f_{-}(\eta)=\eta+\phi(\eta), \phi(\eta+2 \pi)=\phi(\eta),\left|\phi^{\prime}(\eta)\right|<1$ [8]. Однако с их помошью для произвольной мировой поверхности невозможно добиться равенства $\sigma_{3}=$ const (и $\sigma_{0}=$ const) при всех $\tau$. В связи с этим после наложения условий (1.3) и (1.6) возникает необходимость определения трех функций $\sigma_{0}(\tau), \sigma_{3}(\tau)$ и $\tau^{*}(\tau)$, что существенно усложняет, например, решение начально-краевой задачи для данной барионной модели по сравнению с аналогичной задачей для струны, моделирующей мезон [9].

Для рассматриваемых в настояшей работе равномерных врашений системы вид упомянутых трех функций определяется соображениями симметрии.

\section{2. РЕШЕНИЯ, ОПИСЫВАЮШИЕ ПЛОСКИЕ РАВНОМЕРНЫЕ ВРАШЕНИЯ}

В работе [7] было получено решение поставленной задачи, описывающее равномерное врашение системы (из трех материальных точек вместе со связывающей их релятивистской струной) в плоскости $x y$ относительно начала координат. При этом форма замкнутой струны одинакова во все моменты времени. Траектории кварков в $(2+1)$-мерном пространстве Минковского являются винтовыми линиями и заданы внутренними уравнениями $\sigma=\sigma_{i}=$ const, $i=0,1,2,3$. Это решение имеет вид

$$
X^{\mu}=\left\{t_{0}+a \tau+b \sigma ; u(\sigma) \cos \omega \tau-\tilde{u}(\sigma) \sin \omega \tau ; u(\sigma) \sin \omega \tau+\tilde{u}(\sigma) \cos \omega \tau\right\} .
$$

В данное выражение входят константы $a, b, \omega$ и функции $u(\sigma), \tilde{u}(\sigma)$, где

$$
u(\sigma)=\left\{\begin{array}{lll}
A_{0} \cos \omega \sigma+B_{0} \sin \omega \sigma, & \sigma \in\left[\sigma_{0}, \sigma_{1}\right], & \\
A_{1} \cos \omega \sigma+B_{1} \sin \omega \sigma, & \sigma \in\left[\sigma_{1}, \sigma_{2}\right], & A_{1} \equiv A, \\
A_{2} \cos \omega \sigma+B_{2} \sin \omega \sigma, & \sigma \in\left[\sigma_{2}, \sigma_{3}\right], &
\end{array}\right.
$$

а функция $\tilde{u}(\sigma)=\widetilde{A}_{i} \cos \omega \sigma+\widetilde{B}_{i} \sin \omega \sigma, \sigma \in\left[\sigma_{i}, \sigma_{i+1}\right]$, устроена аналогично. Обозначения $\widetilde{A}_{1} \equiv \widetilde{A}, \widetilde{B}_{1} \equiv \widetilde{B}$ также считаем эквивалентными.

Амплитудные множители $A_{i}, \widetilde{A}_{i}, B_{i}, \widetilde{B}_{i}$ выбраны так, что функции $u(\sigma)$ и $\tilde{u}(\sigma)$ непрерывны на $\left[\sigma_{0}, \sigma_{3}\right]$, но могут иметь разрывы производной при $\sigma=\sigma_{1}$ и $\sigma=\sigma_{2}$.

Значения $\sigma_{1}$ и $\sigma_{2}$ без ограничения общности можно выбрать в виде $\sigma_{1}=0, \sigma_{2}=\pi$, как и в (1.6), ниже этот выбор будет подразумеваться везде, если не оговорено противное. Заметим, что после наложения условий (1.6) две другие константы $\sigma_{0}$ и $\sigma_{3}$ задавать произвольным образом нельзя, они будут определены ниже из системы уравнений.

Выражение (2.1) является решением уравнений движения (1.4). Потребуем, чтобы оно удовлетворяло условиям ортонормальности (1.3), условиям замыкания (1.2) и краевым условиям (1.5). Подстановка (2.1) в два краевых условия (1.5) с $i=1,2$ в совокупности с условиями непрерьвности функций $u(\sigma)$ и $\tilde{u}(\sigma)$ при $\sigma=0$ и $\sigma=\pi$ дает по четыре уравнения для $u$ и $\tilde{u}$, позволяюших выразить все $A_{i}, B_{i}$ через $A$ и $B$ (и по этим же формулам - $\widetilde{A}_{i}, \widetilde{B}_{i}$ через $\widetilde{A}$ и $\left.\widetilde{B}\right)$ :

$$
\begin{array}{ll}
A_{0}=A, & A_{2}=\left(1+h_{2} c s\right) A+h_{2} s^{2} B, \\
B_{0}=h_{1} A+B, & B_{2}=-h_{2} c^{2} A+\left(1-h_{2} c s\right) B,
\end{array}
$$


здесь введены обозначения

$$
c \equiv c_{1}=\cos \omega\left(\sigma_{2}-\sigma_{1}\right)=\cos \pi \omega, \quad s \equiv s_{1}=\sin \pi \omega, \quad h_{i}=\frac{\omega m_{i}}{\gamma C_{i}}
$$

где

$$
C_{i}=\left.\sqrt{\dot{X}^{2}}\right|_{\sigma=\sigma_{i}}=\text { const }
$$

Требование вьполнения условий ортонормальности (1.3) для решений (2.1) приводит к системе уравнений

$$
\begin{aligned}
A_{i}^{2}+B_{i}^{2}+\widetilde{A}_{i}^{2}+\widetilde{B}_{i}^{2} & =\left(a^{2}+b^{2}\right) / \omega^{2}, & & i=0,1,2 \\
A_{i} \widetilde{B}_{i}-\widetilde{A}_{i} B_{i} & =a b / \omega^{2}, & i & =0,1,2 .
\end{aligned}
$$

Три уравнения (2.4) независимы, тогда как из уравнений (2.5) в качестве независимого можно оставить только одно, отвечающее $i=1$. Если оно выполнено, то остальные два удовлетворяются автоматически в случае, если имеют место равенства (2.3).

Во введенной указанным образом координатной сети линии $\tau=$ const (не совпадающие с сечениями $t=\mathrm{const})$ не замкнуты, и в условии замыкания (1.2) имеем $\tau^{*} \neq \tau$. Однако вследствие свойств симметрии мировой поверхности относительно поворота и одновременной трансляции вдоль оси $t=x^{0}$ связь между $\tau$ и $\tau^{*}$ достаточно проста: $\tau^{*}=\tau+$ const.

Для того чтобы выражение (2.1) было решением поставленной задачи, осталось потребовать выполнения условия замыкания (1.2) и краевого условия (1.5) на траектории третьего $(i=3)$ кварка. Обозначим через $-\theta / \omega$ константу, фигурирующую в этих условиях:

$$
\tau^{*}=\tau-\theta / \omega, \quad \theta=D \omega b / a, \text { где } \quad D=\sigma_{3}-\sigma_{0} .
$$

Выражение для $\theta$ получено подстановкой $X^{0}=t_{0}+a \tau+b \sigma$ в условие замыкания (1.2). Угол $\theta$ имеет следующий геометрический смысл: если провести на мировой поверхности линию $\tau=$ const, $\sigma_{0} \leq \sigma \leq \sigma_{3}$, то $\theta$ есть разность фаз между началом этой линии (при $\left.\sigma=\sigma_{0}\right)$ и ее концом (при $\sigma=\sigma_{3}$ ) на винтовой траектории третьего кварка.

Подставим выражения (2.1)-(2.3) в краевое условие (1.5) и условие замыкания (1.2) при $\mu=1,2$. Значения функций $u(\sigma), \tilde{u}(\sigma)$ и их производных в точках $\sigma=\sigma_{0}$ и $\sigma=\sigma_{3}$ выразим с помошью (2.3) через $A, B, \widetilde{A}, \widetilde{B}$, например:

$$
u\left(\sigma_{3}\right)=\left[\cos \omega \sigma_{3}-h_{2} c \sin \omega\left(\sigma_{3}-\pi\right)\right] A+\left[\sin \omega \sigma_{3}-h_{2} s \sin \omega\left(\sigma_{3}-\pi\right)\right] B .
$$

В четырех уравнениях (1.2) и (1.5) с $\mu=1,2$, которые должны выполняться при всех $\tau$, выразим $\tau^{*}$ с помощью (2.6) и приравняем подобные члены при $\cos \omega \tau$ и $\sin \omega \tau$. Восемь получившихся равенств попарно совпадают и образуют линейную однородную систему из четырех уравнений с четырьмя неизвестными амплитудами $A, B, \widetilde{A}, \widetilde{B}$, имеющую достаточно громоздкий вид. Для упрошения записи и выявления структуры этой системы обозначим

$$
\begin{aligned}
& \delta_{i}=\sigma_{i+1}-\sigma_{i}, \quad D=\sigma_{3}-\sigma_{0}=\delta_{0}+\delta_{1}+\delta_{2}, \\
& c_{i}=\cos \omega \delta_{i}, \quad s_{i}=\sin \omega \delta_{i}, \quad c_{*}=\cos \omega \sigma_{3}, \quad s_{*}=\sin \omega \sigma_{3}
\end{aligned}
$$


и введем матричные обозначения, в которых данная система будет состоять из двух уравнений (каждое из них - столбец):

$$
M_{1}\left(\begin{array}{c}
A \\
\widetilde{A}
\end{array}\right)=M_{2}\left(\begin{array}{c}
B \\
\widetilde{B}
\end{array}\right), \quad M_{3}\left(\begin{array}{c}
A \\
\widetilde{A}
\end{array}\right)=M_{4}\left(\begin{array}{c}
B \\
\widetilde{B}
\end{array}\right),
$$

здесь

$$
\begin{aligned}
M_{1} & =\left(h_{1} s_{0}-c_{0}\right) E+\left(c_{*}-h_{2} c s_{2}\right) U, \quad M_{2}=-s_{0} E-\left(s_{*}-h_{2} s s_{2}\right) U, \\
M_{3} & =\left[\left(1-h_{1} h_{3}\right) s_{0}+\left(h_{1}+h_{3}\right) c_{0}\right] E+\left(s_{*}+h_{2} c c_{2}\right) U, \\
M_{4} & =\left(h_{3} s_{0}-c_{0}\right) E+\left(c_{*}-h_{2} s c_{2}\right) U, \\
U & =U(\theta)=\left(\begin{array}{cc}
\cos \theta & \sin \theta \\
-\sin \theta & \cos \theta
\end{array}\right),
\end{aligned}
$$

$E$ - единичная матрица. Первое из уравнений (2.7) - следствие условия замыкания (1.2), второе - следствие условия (1.5).

Если учесть взаимную коммутативность матриц $M_{k}$ и исключить из системы $(2.7)$ столбец с $A$ и $\widetilde{A}$ или столбец с $B$ и $\widetilde{B}$, то окажется, что каждый из них аннулируется одной и той же матрицей:

$$
M\left(\begin{array}{c}
A \\
\widetilde{A}
\end{array}\right)=0, \quad M\left(\begin{array}{l}
B \\
\widetilde{B}
\end{array}\right)=0
$$

где $M=M_{1} M_{4}-M_{2} M_{3}=E+U^{2}-F U=(2 \cos \theta-F) U$ (здесь использовано тождество $\left.E+U^{2}(\theta)=2 \cos \theta \cdot U\right)$. Величину

$$
F=2 \cos \omega D-\left(h_{1}+h_{2}+h_{3}\right) \sin \omega D+\sum_{i=0}^{2} h_{i} h_{i+1} s_{i} \sin \omega\left(D-\delta_{i}\right)-h_{1} h_{2} h_{3} s_{0} s s_{2}
$$

$\left(h_{0} \equiv h_{3}\right)$ после введения обозначений

$$
\begin{gathered}
G_{1}=\left(h_{1} s_{0} s-s_{0} c-s c_{0}\right) / s_{2}, \quad G_{2}=\left(h_{2} s s_{2}-s c_{2}-s_{2} c\right) / s_{0} \\
G_{3}=\left(h_{3} s_{0} s_{2}-s_{0} c_{2}-s_{2} c_{0}\right) / s
\end{gathered}
$$

удобно преобразовать к простому виду

$$
F=G_{1}+G_{2}+G_{3}-G_{1} G_{2} G_{3}
$$

Однородные системы (2.8) имеют интересуюшее нас нетривиальное решение тогда и только тогда, когда $\operatorname{det} M=0$, что с учетом $\operatorname{det} U=1$ равносильно равенству

$$
2 \cos \theta=G_{1}+G_{2}+G_{3}-G_{1} G_{2} G_{3} .
$$


При выполнении (2.10) матрица $M$ обращается в нуль. Поэтому в качестве собственного можно выбрать произвольный ненулевой “вектор"

$$
\left(\begin{array}{c}
A \\
\widetilde{A}
\end{array}\right) \text { или }\left(\begin{array}{c}
B \\
\widetilde{B}
\end{array}\right),
$$

в частности, как это сделано в приводимых ниже примерах, можно положить $A=0$ (при этом $\widetilde{A}, B, \widetilde{B}$ отличны от нуля). Этот факт связан с поворотной симметрией в выражениях (2.1), (2.2).

При выполнении условия (2.10) два матричных уравнения системы (2.7) становятся равносильными, и с помошью любого из них можно определить, например, $\widetilde{A}, \widetilde{B}$ по заданным произвольно $A$ и $B$ :

$$
\widetilde{A}=-K \cdot\left(h_{1} A+2 B\right), \quad \widetilde{B}=K \cdot\left(2 H A+h_{1} B\right)
$$

где

$$
K=\frac{s_{0} s\left(G_{2} G_{3}-1\right)}{2 s_{2} \sin \theta}, \quad H=\frac{1+h_{1}^{2} K^{2}}{4 K^{2}}=1+\frac{1}{s}\left(h_{1} c+\frac{h_{1}-h_{2}}{h_{2} s-2 c}\right) .
$$

Подставим эти величины в уравнения (2.4) и (2.5), порождаемые условиями ортонормальности (1.3). После преобразований убедимся, что соотношения (2.4) выполняются только тогда, когда параметры $\omega, \delta_{i}$ и $G_{i}$ связаны следуюшим образом:

$$
\begin{gathered}
\frac{G_{3}-G_{2}}{G_{2} G_{3}-1}=\frac{s c_{0}-s_{0} c}{s_{2}}, \quad \frac{G_{1}-G_{3}}{G_{1} G_{3}-1}=\frac{s_{2} c-s c_{2}}{s_{0}}, \\
\frac{G_{2}-G_{1}}{G_{1} G_{2}-1}=\frac{s_{0} c_{2}-s_{2} c_{0}}{s} .
\end{gathered}
$$

Из этих условий лишш два являются независимыми.

Подставив выражения (2.11) в условия (2.4) и (2.5), выразив с помошью (2.8) параметр $b=a \theta /(D \omega)$ и проведя ряд преобразований с помощью $(2.9)-(2.13)$, получим систему уравнений

$$
\begin{aligned}
a^{2} \theta /\left(D \omega^{3}\right) & =2 K\left(H A^{2}+h_{1} A B+B^{2}\right) \\
a^{2} \omega^{-2}\left[1+(\theta /(D \omega))^{2}\right] & =\left[1+\left(4+h_{1}^{2}\right) K^{2}\right]\left(H A^{2}+h_{1} A B+B^{2}\right) .
\end{aligned}
$$

Исключая из этой системы множители $B^{* 2}=H A^{2}+h_{1} A B+B^{2}$ и $a$, придем к соотношению

$$
\frac{D \omega \theta}{D^{2} \omega^{2}+\theta^{2}}=\frac{4 s_{0} s s_{2}\left(G_{2} G_{3}-1\right) \sin \theta}{4 s_{2}^{2} \sin ^{2} \theta+\left(4+h_{1}^{2}\right) s_{0}^{2} s^{2}\left(G_{2} G_{3}-1\right)^{2}} .
$$

Это уравнение определяет для рассматриваемых движений набор допустимых частот $\omega$, если заданы параметры $G_{i}, \delta_{i}$ и $\theta$. Таким образом, характеризующий движение системы с точностью до масштабного множителя набор параметров $\omega, G_{i}, \delta_{i}$ и $\theta$ определяется посредством решения достаточно сложной системы уравнений (2.9), (2.10), (2.13), (2.15). 
После определения этих величин можно произвольным образом задать амплитудные параметры $A$ и $B$ (в частности, положить $A=0$ ) и с помошњю формул $(2.14),(2.8)$, (2.11), (2.3) найти окончательно вид мировой поверхности (2.1).

\section{3. МИРОВЫЕ ПОВЕРХНОСТИ И ИХ СЕЧЕНИЯ ПЛОСКОСТЯМИ $t=\mathrm{const}$}

Простейшая схема построения мировой поверхности вида (2.1), отвечающей равномерному врашению рассматриваемой системы, предполагает задание параметров (2.9) $G_{1}, G_{2}, G_{3}$ в качестве исходных данных. Следуюшим шагом в этой схеме является определение значения угла $\theta$ по формуле (2.10) и величин $\delta_{i}$ с помошью соотношений $(2.13)$. После подстановки $G_{i}, \delta_{i}$ и $\theta$ в уравнение $(2.15)$ набор допустимых частот $\omega$-корней этого уравнения - может быть найден численно, например с помощью метода секущих [10].

На каждом шаге этой схемы соответствуюшие параметры определяются неоднозначно, например, удовлетворяюшие (2.10) значения $\theta=\theta_{j_{1}}$ образуют счетный набор, нумеруемый дискретным параметром $j_{1}$.

Параметры (2.14) $\delta_{0}$ и $\delta_{2}$ (значение $\delta_{1}=\pi$ зафиксировано без потери общности) находятся с помощью (2.13) по заданным $G_{i}$ так же неоднозначно. Эту процедуру можно реализовать, например, записав правую часть последнего уравнения (2.13) в виде $s^{-1} \sin \omega\left(\delta_{0}-\delta_{2}\right)$. Это уравнение позволяет определить величину

$$
\delta_{02}=\delta_{0}-\delta_{2}=\frac{1}{\omega}\left[(-1)^{j_{2}} \arcsin s \frac{G_{2}-G_{1}}{G_{1} G_{2}-1}+\pi j_{2}\right], \quad j_{2} \in \mathbb{Z} .
$$

При этом появляется дополнительный произвол в выборе дискретного параметра $j_{2}$.

Подстановка $\delta_{2}=\delta_{0}-\delta_{02}$ во второе из уравнений (2.13) дает возможность определить значения

$$
\delta_{0}=\frac{1}{\omega}\left[\operatorname{arctg} \frac{\sin \omega\left(\delta_{02}+\pi\right)}{\cos \omega\left(\delta_{02}+\pi\right)-\left(G_{1}-G_{3}\right) /\left(G_{1} G_{3}-1\right)}+\pi j_{3}\right], \quad j_{3} \in \mathbb{Z},
$$

и $\delta_{2}$ "с точностью" до третьего дискретного параметра $j_{3}$.

Подстановка $G_{i}, \delta_{i}$ и $\theta$ в $(2.15)$ приводит к счетному набору корней этого уравнения - частот $\omega_{l}$.

В результате описанной процедуры возникает набор решений поставленной задачи, различаюшихся в топологическом смысле.

В работе [7] был подробно рассмотрен частный случай, в котором конфигурация системы с тремя одинаковыми кварками симметрична относительно циклических перестановок. При этом для всех трех кварков равны значения $m_{i}, C_{i}, Q_{i}$, а также

$$
\begin{gathered}
G_{1}=G_{2}=G_{3}=G, \\
\delta_{1}=\delta_{2}=\delta_{3}=\pi \Leftrightarrow \sigma_{0}=-\pi, \quad \sigma_{3}=2 \pi .
\end{gathered}
$$

Отметим, что (3.3) следует из (3.4) в силу соотношений (2.13). Обратное, вообще говоря, неверно: при выполнении (3.3) имеют место решения (2.13) с $\omega\left(\delta_{i}-\delta_{j}\right)=\pi k, k \in \mathbb{Z}$, соответствуюшие выбору различных значений $j_{2}$ и $j_{3}$ в $(3.1),(3.2)$. 
Рассмотрим ситуацию, когда соотношения (3.3) и (3.4) не выполнены, в частности случай произвольных масс кварков. Параметры мировой поверхности определяются с помошью описанной выше процедуры. Чтобы иметь представление о соответствуюшем врашательном движении системы, достаточно рассмотреть сечение мировой поверхности плоскостью $t=t_{0}=$ const. Криволинейный треугольник, получающийся в сечении, можно воспринимать как “фотографию” положения струны в момент времени $t$.

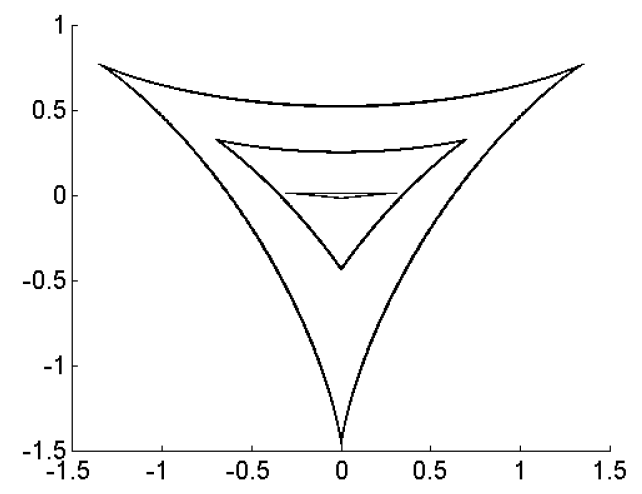

Рис. 1. Простые движения системы с $m_{1}=3, m_{2}=m_{3}=1$.

Характерный вид данных сечений мировой поверхности показан на рис. $1-3$. В целях экономии места на рис. 1, 2 изображено по три кривые, “вложенные” одна в другую за счет выбора амплитуды $B$, на рис. 3 кривые разнесены пространственно.

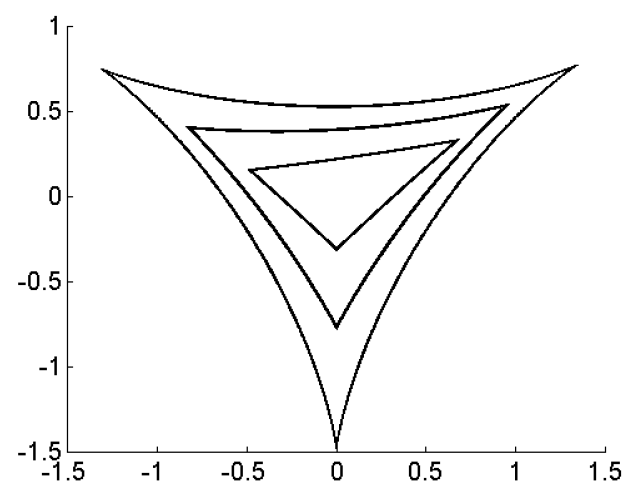

Рис. 2. Простые движения системы с $m_{1}=4, m_{2}=2, m_{3}=3$.

Параметризацию сечений, показанных на рис. $1-3$, приведем для случая $A=0$ (этот 


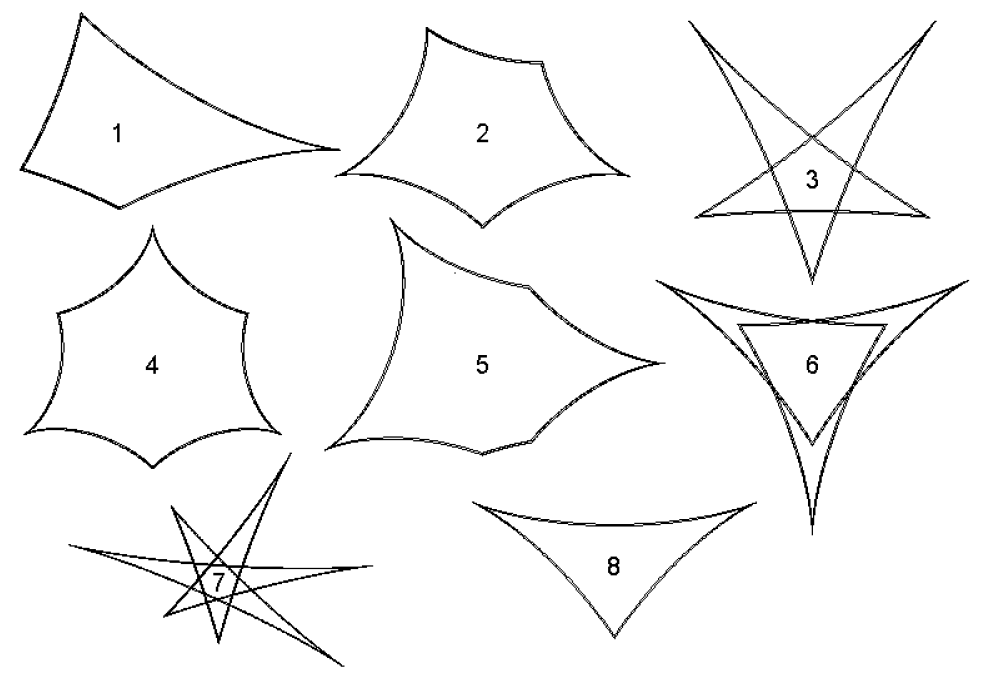

Рис. 3. Примеры экзотических состояний.

выбор влияет на ориентацию, но не на форму кривой) на отрезке $\sigma \in[0, \pi]$ :

$$
\begin{aligned}
& x=B\left[\sin \omega \sigma \cdot \cos \frac{\theta}{D} \sigma+K\left(h_{1} \sin \omega \sigma-2 \cos \omega \sigma\right) \sin \frac{\theta}{D} \sigma\right], \\
& y=B\left[-\sin \omega \sigma \cdot \sin \frac{\theta}{D} \sigma+K\left(h_{1} \sin \omega \sigma-2 \cos \omega \sigma\right) \cos \frac{\theta}{D} \sigma\right] .
\end{aligned}
$$

Величины $D, h_{1}$ и $K$ определяются соответственно формулами (2.6), (2.9) и (2.12).

С помощью (2.3) выражения вида (3.5) можно получить и для двух других сторон криволинейного треугольника. Так, в частности, при $\sigma \in\left[\sigma_{0}, 0\right]$ в $(3.5)$ достаточно заменить $\left(h_{1} \sin \omega \sigma-2 \cos \omega \sigma\right)$ на $\left(-h_{1} \sin \omega \sigma-2 \cos \omega \sigma\right)$.

Изображенные на рис. $1-3$ кривые вращаются в плоскости $x y$ с угловой частотой $\Omega=$ $\omega / a$. Значение параметра $а$ определяется из (2.14):

$$
a=\sqrt{2 D K \omega^{3} \theta^{-1}} B^{*}
$$

амплитудньй множитель $B^{*}=\sqrt{H A^{2}+h_{1} A B+B^{2}}$ в случае $A=0$ равен $|B|$. При этом кварки движутся со скоростями $v_{i}$ по окружностям радиусов $R_{i}$, где

$$
\begin{aligned}
v_{1}=\sqrt{\frac{2 K \theta}{D \omega}} & =\sqrt{\frac{\theta s s_{0}\left(G_{2} G_{3}-1\right)}{D \omega s_{2} \sin \theta}}, & & v_{2}=\sqrt{\frac{\theta s s_{2}\left(G_{1} G_{3}-1\right)}{D \omega s_{0} \sin \theta},} \\
v_{3} & =\sqrt{\frac{\theta s_{0} s_{2}\left(G_{1} G_{2}-1\right)}{D \omega s \sin \theta}}, & R_{i} & =\frac{v_{i}}{\Omega}=\frac{a}{\omega} v_{i} .
\end{aligned}
$$


Напомним, что в выбранных единицах измерения скорость света равна 1.

Ротационное движение (состояние) системы будем называть простым, если сечение мировой поверхности плоскостью $t=$ const является криволинейным треугольником с гладкими сторонами без особенностей. Такие конфигурации показаны на рис. 1 и 2. Движения, не относяшиеся к категории простых (см. рис. 3), будем называть әкзотическими.

Движение системы является простым (рис. 1,2 ) тогда и только тогда, когда выполнены ограничения

$$
|\theta| \leq \pi, \quad G_{i}>1, \quad i=1,2,3, \quad G_{1}+G_{2}+G_{3}-G_{1} G_{2} G_{3} \geq-2,
$$

при этом $\omega$ - минимальный по модулю ненулевой корень (2.15), удовлетворяющий условиям $|\omega| \delta_{i} \leq \pi, i=0,1,2$. В частном случае равенства двух масс, например $m_{2}=m_{3}$, условия (3.8) для $G_{i}$ принимают вид $G_{1}>1,1<G_{2}=G_{3}<1+2 / G_{1}$, в симметричном случае $(3.3)-(3.4): 1<G_{1}=G_{2}=G_{3}<2$.

На рис. 1 и 2 показана зависимость формы криволинейного треугольника от скорости вращения при фиксированных значениях $m_{i}$, которые равны $m_{1}=3, m_{2}=m_{3}=1$ (см. рис. 1$)$ и $m_{1}=4, m_{2}=2, m_{3}=3$ (см. рис. 2 ). В такой постановке исходными данными являются значения масс кварков (исследуются движения одной и той же системы) и один из параметров, связанных со скоростью врашения, например $G_{1}$. Массы $m_{i}$ и другие параметры модели связаны следуюшим образом:

$$
m_{i}=\frac{\gamma}{\omega} C_{i} h_{i}=\frac{\gamma}{\omega} a h_{i} \sqrt{1-v_{i}^{2}}
$$

здесь параметр $а$ определяется формулой (3.6), а $v_{i}$ - формулой (3.7).

Для решения системы нелинейных уравнений (2.9)-(2.15), (3.7), (3.9) используем следующий подход. Наряду с фиксированным $G_{1}$ задаем пробные значения $G_{2}$ и $G_{3}$. На этой основе определяем с помощью (2.10), (2.15), (3.1), (3.2) значения $\theta, \omega$ и $\delta_{i}$. Дискретные параметры $j_{1}, j_{2}, j_{3}$ выбираем в соответствии с исследуемым состоянием. Вычисленные с помошью (3.7), (3.9) значения отношений масс $m_{i} / m_{1}, i=2,3$, с ростом номера итерации $n$ стремятся к заданным отношениям масс. В основу итерационного процесса был положен двумерный вариант метода секущих [10]. Отметим, что данный итерационный метод используется здесь на двух уровнях, причем на первом - при решении системы уравнений $(3.1),(3.2),(2.15)$ с одновременным определением $\omega, \delta_{0}$ и $\delta_{2}$.

На рис. 1 и 2 изображены состояния, характеризуемые различными значениями скорости врашения. Амплитуды $B^{*}$ были выбраны так, что размер малых внутренних треугольников в несколько раз увеличен по сравнению с естественным (чем выше скорости $v_{i}$, тем больше размер треугольника), а размер внешних в несколько раз уменьшен. Естественным здесь назван размер или масштабный фактор $B_{n}^{*}$, определяемый формулами (3.9), (3.6) и отвечающий разным движениям одной и той же системы с теми же $m_{i}$ и $\gamma$. Для средних треугольников на рис. 1 и 2 имеем $\gamma=1$, для остальных естественный масштаб не сохранен из-за сильной зависимости размеров $R_{i}$ от скорости врашения. Значения $G_{1}, G_{2}$, минимальной $v_{1}$ и максимальной $v_{2}$ скоростей, коэффициента 
увеличения $B / B_{n}$ и другие для простых движений на рис. 1 и 2 приведены ниже (первые столбцы описывают внутренние треугольники):

\begin{tabular}{|c|c|c|c|c|c|c|c|c|}
\hline \multicolumn{5}{|c|}{ К рис. $1 \quad\left(m_{i}=3,1,1\right)$} & \multicolumn{4}{|c|}{ К рис. $2 \quad\left(m_{i}=4,2,3\right)$} \\
\hline$G_{1}$ & $=$ & 1.61 & 2.3 & 2.7 & $G_{1}$ & $=1.15$ & 1.7 & 2.2 \\
\hline$G_{2}$ & $\simeq$ & 1.0023 & 1.31 & 1.68 & $G_{2}$ & $\simeq 1.041$ & 1.312 & 1.69 \\
\hline$\omega$ & $\simeq$ & 0.168 & 0.387 & 0.77 & $\omega$ & $\simeq 0.143$ & 0.386 & 0.75 \\
\hline$\theta$ & $\simeq$ & 0.053 & 1.063 & 2.46 & $\theta$ & $\simeq 0.139$ & 0.923 & 2.328 \\
\hline$v_{1}$ & $\simeq$ & 0.025 & 0.433 & 0.908 & $v_{1}$ & $\simeq 0.145$ & 0.497 & 0.91 \\
\hline$v_{2}$ & $\simeq$ & 0.503 & 0.768 & 0.97 & $v_{2}$ & $\simeq 0.354$ & 0.711 & 0.954 \\
\hline$\delta_{2}$ & $\simeq$ & 6.25 & 4.38 & 3.392 & $\delta_{2}$ & $\simeq 3.955$ & 3.493 & 3.222 \\
\hline $3 / B_{n}$ & $=$ & 1.7 & 1 & 0.19 & $B / B_{n}$ & $=$ & 1 & $1 / 7$ \\
\hline
\end{tabular}

При уменьшении скоростей кварков $v_{i}$, значений параметров $\omega$ и $\theta$ криволинейный треугольник стремится к прямолинейному. Предельная форма последнего зависит от того, удовлетворяют ли значения масс $m_{i}$ неравенству треугольника. Если одна из масс превышает сумму двух других, например $m_{1}>m_{2}+m_{3}$ (см. рис. 1 ), то в пределе $\theta \rightarrow 0$ величина тупого угла в вершине, соответствуюшей максимальной из масс $m_{1}$, стремится к $\pi$, а треугольник по форме приближается к отрезку. Предел $\theta=0, R_{1}=0, v_{1}=0$ (при этом $\delta_{2}=\delta_{0}+\delta_{1}, G_{2}=G_{3}=1+0, G_{1}>1$ ) достигается при конечных значениях $\omega, v_{2}=s, v_{3}=s_{0}$. При дальнейшем уменьшении скоростей $v_{2}$ и $v_{3}$ тяжелый кварк оказывается в центре вращения, а струна сохраняет форму отрезка. Данная конфигурация выглядит как врашение нагруженной тяжелым кварком прямолинейной релятивистской струны с натяжением $2 \gamma$, моделирующей мезон $[1,4,11]$.

Если неравенство треугольника для $m_{i}$ выполнено (см. рис. 2), то в нерелятивистском пределе для величин $(2.9)$ имеем $G_{i} \rightarrow 1+0$, а длины сторон $\ell_{i j}$ (между кварками $m_{i}$ и $m_{j}$ ) прямолинейного треугольника пропорциональны как соответствующим $\delta_{i}$, так и массам в противоположных вершинах:

$$
\frac{\ell_{12}}{\delta_{1}}=\frac{\ell_{23}}{\delta_{2}}=\frac{\ell_{31}}{\delta_{0}}, \quad \frac{\delta_{1}}{m_{3}}=\frac{\delta_{2}}{m_{1}}=\frac{\delta_{0}}{m_{2}} \quad\left(\omega \rightarrow 0, \quad v_{i} \rightarrow 0\right) .
$$

В противоположном ультрарелятивистском пределе $v_{i} \rightarrow 1$ для простых состояний величины $\delta_{0}$ и $\delta_{2}$ стремятся к $\delta_{1}=\pi,|\omega| \rightarrow 1-0,|\theta| \rightarrow \pi-0$, а криволинейный треугольник вне зависимости от значений $m_{i}$ стремится по форме к трехзвенной гипоциклоиде

$$
x=B\left(2 \sin \frac{2}{3} \sigma-\sin \frac{4}{3} \sigma\right), \quad y=-B\left(2 \cos \frac{2}{3} \sigma+\cos \frac{4}{3} \sigma\right), \quad \sigma \in[-\pi, 2 \pi]
$$

(гипоциклоида - траектория точки окружности, катящейся внутри неподвижной окружности большего радиуса [12]). В случае (3.11) отношение этих радиусов равно 3 , такую гипоциклоиду назьвают дельтоидой.

Предельный переход $v_{i} \rightarrow 1$ или $m_{i} \rightarrow 0$ к (3.11), а также движения замкнутых безмассовых струн, являющиеся врашениями гипоциклоид, рассмотрены в разделе 4 . 
Криволинейные треугольники, изображенные на рис. 1-3, также составлены из фрагментов гипоциклоиды. Отметим, что линия с параметризацией (3.5) при $\sigma \in \mathbb{R}$ является гипоциклоидой тогда и только тогда, когда параметры $\omega, \theta, D$ и $G_{i}$ связаны условием (2.15). Для этой гипоциклоиды отношение радиусов окружностей 2(1 $|b| / a)^{-1}=2(1-|\theta /(D \omega)|)^{-1}$ не обязательно рационально.

Классифицируя движения системы, отметим, что замена $\theta$ на $-\theta$ меняет направление обхода криволинейного треугольника при изменении $\sigma$ от $\sigma_{0}$ до $\sigma_{3}$, а замена $\omega$ на $-\omega$ меняет направление врашения (если $\theta$ и $\omega$ удовлетворяют (2.15), то этому уравнению удовлетворяют и $-\theta$, и $-\omega)$.

Перейдем к рассмотрению экзотических состояний системы, примеры которых приведены на рис. 3. Аналогичные состояния имеют место и для струны, моделируюшей мезон $[8,11]$. Они характеризуются наличием внутренних (безмассовых) точек струны, движушихся со скоростью света. На мировых линиях этих точек метрика имеет особенности вида $\dot{X}^{2}=X^{\prime 2}=0$. На рис. 3 эти особенности, являюшиеся точками возврата гипоциклоид, не следует путать с материальными точками (положениями кварков). В последних фрагменты струны соединены под ненулевым углом, тогда как в особых точках соответствующий угол равен нулю. На рис. 3 (а также рис. 1 и 2) кварк под номером 1 занимает нижнее положение, т.е. имеет координаты $x=0, y<0$, определяемые выбором $A=0, B>0, t-t_{0}=0$. Кварки с номерами 2 и 3 расположены в порядке обхода струны против часовой стрелки. В центре врашения - номер кривой.

Исходными данными для построения представленных кривых служат значения $G_{i}$ и выбранные каким-либо образом $j_{i}$ и $l$ в $(3.1),(3.2),(2.15)$. В симметричном случае (3.3), (3.4) число особенностей $\dot{X}^{2}=0$ на сторонах криволинейного “треугольника" одинаково (кривые 4, 6 и 8 на рис. 3), если же (3.4) не выполнено, возможны конфигурации с различным числом этих особенностей, технически реализуемые с помощью выбора параметров $j_{2}$ и $j_{3}$ в формулах (3.1) и (3.2).

Введем для описания типа состояния системы символ $\left(n_{1}, n_{2}, n_{3}\right)$, где $n_{1}$ - число особенностей на отрезке струны между 1 -м и 2-м кварками, $n_{2}-$ между 2 -м и 3 -м, $n_{3}-$ между 3 -м и 1-м. Эта характеристика неполна, топологически различные кривые 2 и 3 на рис. 3 имеют один и тот же тип $(1,0,1)$ (то же относится и к кривым 4 и 6 типа $(1,1,1)$ ).

Опишем кратко изображенные на рис. 3 кривые. Четырехугольная кривая 1 представляет простейшее экзотическое состояние типа $(1,0,0)$ с одной особенностью и отвечает значениям $G_{1}=0.2, G_{2}=-0.4, G_{3}=-0.2, \omega \simeq 1.23, \theta \simeq 1.78, j_{i}=0$. Соответствующие значения масс кварков следующие: $m_{1} \simeq 3.04, m_{2} \simeq 2.59, m_{3} \simeq 3.36$ при $\gamma=1$. Пятиугольная линия 2 характеризуется значениями $G_{1}=0.2, G_{2}=0.1$, $G_{3}=0.5, \theta \simeq 5.12$; звездообразная пятилучевая конфигурация 3 - значениями $G_{1}=1.9$, $G_{2}=G_{3}=1.7, v_{1} \simeq 0.534, v_{2}=v_{3} \simeq 0.6, \omega \simeq 1.37, \theta \simeq 1.67, j_{1}=0, j_{2}=1$ и $j_{3}=1$ (в точках самопересечения различные отрезки струны вида (1.1) не взаимодействуют).

Кривые 4-6 на рис. 3 представляют различные экзотические состояния системы с одинаковыми массами кварков $m_{1}=m_{2}=m_{3}$, удовлетворяющие условию (3.3). При этом кривые 4 и 6 описывают симметричные (условие (3.4) выполнено) движения типа $(1,1,1)$ различной конфигурации. Симметричный криволинейный шестиугольник 4

5 Теоретическая и математическая физика, т. 114, № 2, 1998 г. 
характеризуется значениями $G_{1}=G_{2}=G_{3}=G=0, \theta=5 \pi / 2, \omega \simeq 1.35$. У этой кривой "массивные" вершины (с кварками, движушимися со скоростью $v \simeq 0.742$ ) чередуются с безмассовыми, движушимися со скоростью света. Кривая 6 с самопересечениями определяется значениями $G=-1, \theta=\pi, \omega \simeq 1.27$. Кривая 5 имеет тип $(0,1,2)$, соответствует одинаковым значениям $G_{1}=G_{2}=G_{3}=1.5, m_{1}=m_{2}=m_{3} \simeq 0.4$ и иллюстрирует недостаточность выполнения условия (3.3) для симметричности движения. Величины $\delta_{0} \simeq 17.7 \pi, \delta_{2} \simeq 9.4 \pi, \omega \simeq 0.12$ и $\theta \simeq 5.31$ в этом случае обеспечивают различное число особенностей на сторонах "треугольника".

Семиугольная звездчатая кривая 7 имеет тип $(1,3,0)$ и характеризуется значениями $G_{1}=G_{2}=2, G_{3}=1.5, \omega \simeq 1.27, \theta \simeq 1.82, j_{2}=-3$.

Криволинейный "треугольник" 8 соответствует симметричному решению с $G=0.9$, $\theta \simeq 6.11, \omega \simeq 2.276$ и имеет по две особенности на каждой из струн, соединяюших кварки. При изменении $\sigma$ от $-\pi$ до $2 \pi$ эта замкнутая кривая обходится трижды, три кварка совмешены в нижней из вершин, каждый отрезок данной кривой образован тремя совмешенными струнами.

\section{4. КЛАССИФИКАЦИЯ ТОПОЛОГИЧЕСКИХ ТИПОВ РОТАЦИОННЫХ ДВИЖЕНИЙ}

Перейдем к упорядочению представленного в предыдушем разделе обширного набора различных в топологическом смысле состояний врашаюшейся релятивистской струны, нагруженной тремя материальными точками. В основу классификации положим исследование предельных конфигураций мировых поверхностей и их сечений $t=$ const в случае, когда массы кварков стремятся к нулю. При непрерывном изменении значений масс $m_{i}$ рассматриваемое движение остается в рамках одного и того же топологического класса. В пределе $m_{i} \rightarrow 0$ каждому такому классу будет соответствовать некоторое ротационное движение (врашение) замкнутой безмассовой релятивистской струны, имеющей, как будет показано ниже, форму гипоциклоиды.

Рассмотрим предельный переход $m_{i} \rightarrow 0$. Анализ выражений (2.9)-(2.15), (3.7), (3.9) показывает, что в этом пределе независимо от отношений масс $m_{i}$ параметры простого или экзотического состояния типа $\left(n_{1}, n_{2}, n_{3}\right)$ стремятся к следуюшим значениям: $v_{i} \rightarrow 1, h_{i} \rightarrow 0,|\omega| \delta_{i} \rightarrow \pi\left(1+n_{i}\right)-0,|\theta| \rightarrow \pi k-0, k$ - целое. Введем на мировой поверхности (2.1) новые координаты $\tau, \sigma$ (отличаюшиеся от старых домножением на константу $\left.1+n_{1}\right)$, в которых условие (1.6) заменено более обшим: $\sigma_{2}=\delta_{1}=\pi\left(1+n_{1}\right)$. В таких координатах предел $m_{i} \rightarrow 0$ соответствует $\omega \rightarrow 1-0$. Вводя малые отклонения величин $\omega, \theta, \delta_{0}, \delta_{2}$ от предельных значений

$$
\omega=1-\varepsilon, \quad \delta_{0}=\pi\left(1+n_{3}\right)-\Delta_{0}, \quad \delta_{2}=\pi\left(1+n_{2}\right)-\Delta_{2}
$$

и подставляя эти выражения в $(2.9)-(2.15),(3.7)$, находим, что при $m_{i} \rightarrow 0$

$$
\theta=\pi k\left(1-\varepsilon-\frac{\Delta_{0}+\Delta_{2}}{\pi n}\right), \quad \lim _{m_{i} \rightarrow 0} 2 K=\frac{n}{k}
$$


С учетом (2.11) приходим к следуюшему предельному выражению для мировой поверхности (2.1), описываюшему все отрезки струны:

$$
X^{\mu}=\widetilde{B}\{n \tau+k \sigma ; k \sin \sigma \cos \tau+n \cos \sigma \sin \tau ; k \sin \sigma \sin \tau-n \cos \sigma \cos \tau\} .
$$

Здесь $\sigma \in[0, \pi n]$, зафиксирована “калибровка" $A=0$, а целочисленные параметры $n$ и $k$, фигурируюшие в (4.2) и (4.3), определяются и ограничены в своих значениях следующим образом:

$$
n=\lim _{m_{i} \rightarrow 0} \omega \frac{D}{\pi}, \quad k=\lim _{m_{i} \rightarrow 0} \frac{\theta}{\pi}, \quad n \geq 3, \quad|k| \leq n-2, \quad n-k \text { четно. }
$$

В частности, для простых состояний имеем $n=3,|k|=1$; сечение соответствуюшей поверхности (4.3) плоскостью $t=$ const - дельтоида вида (3.11).

Эти поверхности с точностью до масштабирования и движений в пространстве Минковского определяются двумя параметрами $n$ и $k$ и заметаются врашающейся релятивистской струной, имеющей форму гипоциклоиды [12]

$$
\begin{aligned}
& x=B\left(k \sin \sigma \cos \frac{k}{n} \sigma-n \cos \sigma \sin \frac{k}{n} \sigma\right), \\
& y=-B\left(k \sin \sigma \sin \frac{k}{n} \sigma+n \cos \sigma \cos \frac{k}{n} \sigma\right), \quad \sigma \in[0, \pi n],
\end{aligned}
$$

с $n$ точками возврата $\sigma=\pi j$, которые движутся со световой скоростью. Гипоциклоида (4.5), являющаяся сечением поверхности (4.3) плоскостью $t=$ const, характеризуется рациональным отношением радиусов неподвижной и подвижной окружностей

$$
\frac{R}{r}=\frac{2 n}{n-|k|}
$$

В случае $|k|=n-2$ это отношение равно $n$ и кривая не имеет самопересечений. Случаю $|k| \leq n-4$ соответствует звездообразная гипоциклоида с самопересечениями.

Числа особенностей на сторонах “треугольника" $n_{1}, n_{2}, n_{3}$ удовлетворяют предельным соотношениям

$$
n_{1}+1=\lim _{m_{i} \rightarrow 0} \omega \delta_{1} / \pi, \quad n_{2}+1=\lim _{m_{i} \rightarrow 0} \omega \delta_{2} / \pi, \quad n_{3}+1=\lim _{m_{i} \rightarrow 0} \omega \delta_{0} / \pi
$$

Отсюда следует связь между значением параметра $n$, определенного в $(4.4)$, и числом особенностей $n_{1}+n_{2}+n_{3}$ исследуемого состояния системы:

$$
n=n_{1}+n_{2}+n_{3}+3
$$

Это означает, что в ультрарелятивистском пределе $v \rightarrow 1$ или $m_{i} \rightarrow 0$ состояние типа $\left(n_{1}, n_{2}, n_{3}\right)$ стремится к врашаюшейся гипоциклоиде вида (4.5), причем к $n_{1}+n_{2}+n_{3}$ особым точкам добавляются три бывшие "массивные". 
Отметим, что мировые поверхности (4.3) можно связать, абстрагировавшись от исходной модели, с движениями замкнутой безмассовой релятивистской струны. Нетрудно проверить, что в рамках этой модели выражение (4.3) является решением уравнения (1.4), удовлетворяет условиям ортонормальности (1.3) и условию замыкания вида $(1.2) X^{\mu}(\tau, 0)=X^{\mu}(\tau-\pi k, \pi n)$ при $n \geq 2$ и указанных в (4.4) значениях $k$.

Параметр $k$ в (4.3)-(4.5) может принимать значения $n-2, n-4, \ldots,-(n-2)$, однако состояния, получаюшиеся заменой $k$ на $-k$, отличаются лишь направлением обхода кривой (4.5) и должны быть отнесены к одному топологическому типу. Это связано с инвариантностью типа движения (2.1) относительно замены $\theta$ на $-\theta$. Топологические типы с совпадающими значениями $n$, но различными $k \geq 0$ различны.

Состояния системы с одинаковыми числами $n_{1}, n_{2}, n_{3}, k$, а также в силу (4.7) и $n$ относятся к одному и тому же топологическому типу. Напротив, состояния относятся к различным топологическим типам, если у них не совпадают значения $n$, или $k \geq 0$, или соответствуюшие тройки $n_{1}, n_{2}, n_{3}$ не получаются друг из друга перестановкой, которая эквивалентна перенумерации кварков.

Таким образом, для исчерпывающей классификации типов ротационных движений исследуемой системы достаточно указать все возможные наборы целочисленных параметров $\left(n, k ; n_{1}, n_{2}, n_{3}\right)$, удовлетворяющих перечисленным выше условиям. Удобно указывать все пять параметров, имея в виду, что в силу (4.7) можно ограничиться четырьмя, опустив $n$ или один из $n_{i}$.

Порядок перечисления топологических типов может быть следующим. Выбираем $n \geq 3$, затем неотрицательное значение $k$ из набора $n-2, n-4, \ldots$ - всего $[n / 2]$ возможных значений $k$ для данного $n$. Далее следует выбрать любым способом три точки возврата гипоциклоиды (4.5) и “поместить" в них точечные массы $m_{1}, m_{2}, m_{3}$ (порядок их размешения в данном случае не важен, т.к. в определение топологического типа была заложена возможность непрерывного изменения масс кварков). Это равносильно выбору тройки чисел $\left(n_{1}, n_{2}, n_{3}\right)$. Тем самым будет зафиксирован один из топологических типов состояния системы.

Применим введенную здесь классификацию к примерам, рассмотренным в предыдущем разделе. Простые движения, показанные на рис. 1 и 2, очевидно, характеризуются набором $(3,1 ; 0,0,0)$. Экзотические состояния на рис. 3 имеют следующие типы: 1 $(4,2 ; 1,0,0), 2-(5,3 ; 1,0,1), 3-(5,1 ; 1,0,1)$ (для случая $n=5$ представлены оба возможных значения $k=1$ и $k=3), 4-(6,4 ; 1,1,1), 5-(6,4 ; 0,1,2), 6-(6,2 ; 1,1,1)$, $7-(7,1 ; 1,3,0)$ и $8-(9,3 ; 2,2,2)$. Состояния 6 и 8 в пределе $m_{i} \rightarrow 0$ переходят в гипоциклоиду (4.5), имеющую вид дельтоиды, которая, однако, обходится соответственно два и три раза. Эти примеры подчеркивают тот факт, что для классификации топологических типов состояний задавать отношение радиусов (4.6) или, что эквивалентно, отношение $k / n$ недостаточно. Следует указьвать оба параметра $k$ и $n$.

В то же время движения, отвечаюшие значению $k=0$ и имеющие место при четных $n=4,6,8, \ldots$, на рис. $1-3$ не показаны. Они устроены достаточно просто: система имеет вид равномерно врашающегося прямолинейного отрезка, составленнного из $n$ отрезков сложенной $n$ раз замкнутой струны. Кварки и "безмассовые" особенности $\dot{X}^{2}=0$ на- 
ходятся в точках сгиба. В этом случае в уравнении $(2.1) b=\theta=0, \tilde{u}(\sigma)=u(\sigma) \cdot$ const. Состояния данного вида имеют близкие аналоги в струнной модели мезона - врашения сложенной $n$ раз открытой струны с массами на концах $[8,11]$.

Завершая классификацию топологических типов ротационных движений в модели “треугольник”, отметим возможность существования прямолинейных конфигураций с одним или более кварками, покоящимися в центре врашения. Пример такого движения - предел при $v_{1} \rightarrow 0$ или $R_{1} \rightarrow 0$ на рис. 1 . В этом случае при анализе следует, исключив покоящиеся кварки, заменить систему более простой и описывать ее предельное состояние тем же выражением (4.3) с $n \geq 2, k=0$.

\section{5. ЭНЕРГИЯ И МОМЕНТ РАССМАТРИВАЕМЫХ СОСТОЯНИЙ}

В данном разделе коротко обсудим возможность приложения полученных решений для струнной модели “треугольник" к описанию барионных состояний, лежаших на некоторой траектории Редже. Эти состояния являются частицами с одинаковым кварковым составом, у которых совпадают почти все квантовые числа [13].

Траектория Редже представляет собой линейную зависимость (не имеющую пока удовлетворительного теоретического объяснения) $J=\alpha^{\prime} E^{2}+\alpha_{0}$ между квадратом массы или энергии покоя $M^{2}=E^{2}$ частицы и ее спином $J$.

В работе [7] для струнной модели бариона “треугольник” на классическом уровне были найдены выражения для энергии

$$
E=\gamma D \frac{a^{2}-b^{2}}{a}+\sum_{i=1}^{3} \frac{m_{i}}{\sqrt{1-v_{i}^{2}}}=\gamma D a\left[1-\left(\frac{\theta}{D \omega}\right)^{2}\right]+\sum_{i=1}^{3} \frac{m_{i}}{\sqrt{1-v_{i}^{2}}}
$$

и углового момента

$$
J=\int_{\sigma_{0}}^{\sigma_{3}}\left(X P_{y}-Y P_{x}\right) d \sigma=\frac{a}{2 \omega}\left(E-\sum_{i=1}^{3} m_{i} \sqrt{1-v_{i}^{2}}\right)
$$

состояния (2.1). Параметры $D, b, a$ и $v_{i}$ определяются соответственно формулами (2.6), (3.6) и (3.7). Соотношение между $E, J$ и частотой вращения $\Omega=\omega / a$ имеет практически такой же вид, как и в струнной модели мезона [4].

Выражения (5.1) и (5.2) неявным образом задают нелинейную связь между энергией и моментом рассматриваемой системы. Вид этой связи определяется не только непрерывными параметрами $\Omega, \omega$ или $\theta$, характеризующими скорость вращения, но и топологическим типом $\left(n, k ; n_{1}, n_{2}, n_{3}\right)$ данного состояния системы. При изменении топологического типа величины $E$ и $J$ меняются скачкообразно.

На рис. 4 представлены результаты численных расчетов зависимости $J$ от $E^{2}$ для различных состояний системы с $m_{2}=m_{3}=1, \gamma=1$. Кривые 1,2 и 3 описывают простые движения системы соответственно с $m_{1}=0.2, m_{1}=1$ и $m_{1}=3$, кривые 4 и 5 - экзотические движения в случае одинаковых масс кварков $m_{1}=m_{2}=m_{3}=1$. Кривая 4 соответствует состоянию типа $(4,2 ; 1,0,0)$ (“четырехугольник" 1 на рис. 3 ), а кривая 5 - состоянию типа $(6,2 ; 1,1,1)$ (линия 6 на рис. 3 ). На кривой 3 , которая представляет 


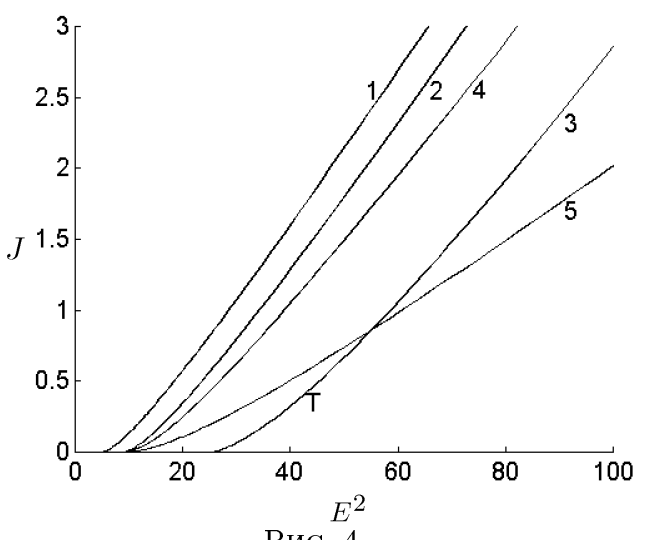

Рис. 4

ту же систему, что и рис. 1 , символом “Т” отмечена точка перехода системы из состояния криволинейного треугольника в состояние вращаюшегося прямолинейного отрезка с натяжением $2 \gamma$ и массой $m_{1}$, покояшейся в центре вращения. Эта точка характеризуется обрашением в нуль параметров $R_{1}, v_{1}, \theta$; тип движения системы в ней меняется с $(3,1 ; 0,0,0)$ на $(2,0 ; 0,0,0)$. Как видим, характер зависимости $J$ от $E^{2}$ в этой точке качественно не меняется.

Исследуем асимптотики соотношения между $E$ и $J$. В ультрарелятивистском пределе $v_{i} \rightarrow 1, E \rightarrow \infty, J \rightarrow \infty$ зависимость $J$ от $E^{2}$ для состояния произвольного типа $\left(n, k ; n_{1}, n_{2}, n_{3}\right)$ стремится к виду, близкому к стандартному:

$$
J \simeq \alpha^{\prime} E^{2}+\alpha_{1} E^{0.5}, \quad \alpha^{\prime}=\frac{1}{2 \pi \gamma} \frac{n}{n^{2}-k^{2}}, \quad v_{i} \rightarrow 1,
$$

причем коэффициент наклона реджевских траекторий отличается от значения Намбу $\alpha^{\prime}=1 /(2 \pi \gamma)$ множителем $n /\left(n^{2}-k^{2}\right)$. Для простых движений этот множитель достигает максимального при допустимых $n$ и $k$ значения $3 / 8$. Следующее из экспериментальных данных примерное равенство наклонов мезонных и барионных траекторий Редже [13] приводит к требованию приблизительно вдвое меньшего значения эффективного натяжения $\gamma$ в барионной модели “треугольник" по сравнению со струнной моделью мезона [4].

Асимптотика (5.3) может быть получена с помощью (4.1). При $\varepsilon \rightarrow 0$ или $v_{i} \rightarrow 1$ первое слагаемое в (5.1) пропорционально $\varepsilon^{-2}$, второе $-\varepsilon^{-1}$, следовательно, первое доминирует. Другими словами, доля в (5.1) кинетической энергии кварков при $v_{i} \rightarrow 1$ стремится к нулю, и это относится к произвольным состояниям системы.

Нерелятивистский предел для простых движений характеризуется неравенствами $v_{i} \ll 1,|\omega| \ll 1,|\theta| \ll 1$. Используя малый параметр $\omega$ в выражениях (5.1), (5.2), (3.6) , (3.7), (3.9), найдем в случае, когда справедливы отношения (3.10), асимптотику

$$
J \simeq q\left(E-\sum_{i=1}^{3} m_{i}\right)^{3 / 2},
$$

где $q=(2 / 3)^{3 / 2} \gamma^{-1} \sqrt{m_{1} m_{2} m_{3}} /\left(m_{1}+m_{2}+m_{3}\right), v_{i} \ll 1$. 


\section{ЗАКЛЮЧЕНИЕ}

В настоящей работе семейство ротационных движений системы исследовано на классическом уровне. Трудности в переходе к квантованию имеют место и для мезонной модели релятивистской струны с массами на концах $[1,8]$. Возможно, разработка барионной модели поможет преодолеть эти трудности.

Приложение полученных в разделах 2 и 3 решений к описанию барионных состояний требует более детального анализа, выходящего за рамки поставленной здесь задачи. В частности, сравнение с экспериментальными данными выражений (5.1)-(5.3) и их близких аналогов в струнной модели мезона [4] открывает возможность определения модельно зависимых значений масс кварков $m_{i}$ и других параметров. В данной работе эта цель не ставилась, поэтому выбор значений $m_{i}$ и $\gamma$ для графиков на рис. 4 был продиктован лишь соображениями иллюстративности.

\section{Список литературы}

[1] Б. М. Барбашов, В. В. Нестеренко. Модель релятивистской струны в физике адронов. М.: Энергоатомиздат, 1987.

[2] A. Chodos, C. B. Thom. Nucl. Phys. B. 1974. V. 72. № 3. P. 509.

[3] В. В. Нестеренко. ТМФ. 1987. Т. 71. № 2. С. 238.

[4] B. M. Barbashov. Classical dynamics of rotating relativistic string with massive ends: the Regge trajectories and quark masses. In: Strong Interactions at Long Distances. Palm Harbor: Hadronic Press, 1995. P. 257.

[5] X. Artru. Phys. Rep. C. 1983. V. 97. № 2, 3. P. 147.

[6] М.С. Плющай, Г.П. Пронько, А. В. Разумов. ТМФ. 1985. Т. 63. № 1. С. 97-112; С.В. Клименко, В.Н. Кочин, М. С. Плющай, Г. П. Пронько, А.В. Разумов, А. В. Самарин. ТМФ. 1985. Т. 64. № 2. С. 245.

[7] Г. С. Шаров. ТМФ. 1997. Т. 113. № 1. С. 68.

[8] Г. С. ШІаров. ТМФ. 1996. Т. 107. № 1. С. 86.

[9] Б. М. Барбашов, Г. С. Шаров. ТМФ. 1994. Т. 101. № 2. С. 253.

[10] Джс. Ф. Трауб. Итерационные методы решения уравнений. М.: Мир, 1985.

[11] В. П. Петров, Г. С. Шаров. ТМФ. 1996. Т. 109. № 2. С. 187.

[12] В. Ю. Ровенский. Теория кривых. Красноярск: КГПУ, 1996.

[13] С. Огава, С. Савада, М. Накагава. Составные модели элементарных частиц. М.: Мир, 1983. 